\title{
Antifragility Amid the COVID-19 Crisis Making healthcare systems thrive through generic organisational skills
}

Nasser Hammad Al-Azri

$$
\begin{aligned}
& \text { الصلابة وسط أزمة كوفيد-19 } \\
& \text { جعل أنظمة الرعاية الصحية تزدهر من خلال المهارات التنظيمية الشاملة }
\end{aligned}
$$

ناصر حماد العزري

$\mathrm{D}$ ISASTERS AND CRISES ARE DISRUPTIVE to the structures and functions of communities. However, the challenges they present also bring opportunities for learning and changing at both individual and organisational levels. Healthcare systems owe much of their developments and advancements to crisis situations. Hence, healthcare organisations need to pay attention to these opportunities for thrivingand not just surviving - amid the chaos of adversities. Thriving through and within crises requires systems to be antifragile.

Taleb coined the term "antifragility" to describe systems that "benefit from shocks" and "thrive and grow when exposed to volatility, randomness, disorder, and stressors and love adventure, risk, and uncertainty". Taleb advances the notion that "by grasping the mechanisms of antifragility we can build a systematic and broad guide to non-predictive decision making under uncertainty in business, politics, medicine, and life in general". ${ }^{1}$ In other words, antifragile systems need to be built and designed so they not only survive during a crisis but thrive through it.

The current global outbreak of COVID-19 presents exceptional challenges that can serve as an opportunity for healthcare systems to thrive and boost their antifragility as the pandemic continues to spread and evolve over time. This makes the current time a valuable chance for healthcare systems to develop their organisational skills proactively in tandem with the development of the crisis itself.

This opportunity to thrive, however, should not be taken for granted. Instead, healthcare systems and organisations need to be prepared, guided and directed towards this thriving learning mode during the crisis. Unfortunately, many healthcare systems are ill-prepared for pandemic crisis management and are not prepared at all for the opportunity to thrive under such circumstances. In 2019, both the Global Preparedness
Monitoring Board and the Global Health Security Index reported a global lack of preparedness for health emergencies worldwide., ${ }^{2,3}$ During a pandemic, health systems are typically absorbed in managing the chaos that ensues and these systems find themselves exhausted afterwards. Hence, they may lose a great opportunity to thrive amid the chaos of a crisis. It is the responsibility of healthcare leaders to guide their systems through the transition of surviving the crisis to thriving in it.

\section{Healthcare Organisational Skills}

Much like individuals, organisations have mechanisms and skills that allow them to survive and thrive. Living organisms might survive with the provision of minimal requirements for living such as breathing, eating and drinking. However, these organisms need more proactive efforts for thriving and growing. Each living organism has its own mechanisms for surviving and thriving. Similarly, organisations and systems can survive with the provision of their basic requirements but thriving requires planned proactive efforts that best suit the organisation.

There are two types of organisational skills used during crises: specific and generic. ${ }^{4}$ On the one hand, specific skills are needed to manage a particular crisis, such as the development of management protocols and measures to manage the outbreak of COVID-19. Hence, specific skills can be viewed as the 'survival skills' necessary to allow the system to continue functioning despite the crisis. On the other hand, generic skills are necessary for the organisation to carry out its functions and duties to achieve its goals and are usually applied to routine duties. These generic skills are also the platform for applying specific skills during a crisis. Generic skills include aspects such as 
leadership, teamwork and communication, and they can enhance the antifragility of the system.

Although generic organisational skills are not specific to a single type of crisis, they are critical for initiating, building and maintaining specific organisational capabilities before as well as during a crisis. Moreover, because they are applicable to routine duties, generic skills remain relevant after the crisis. If these skills are well developed, they can help an organisation thrive well beyond the crisis, adding to the system's reservoir of antifragility mechanisms.

Much of the current literature concerning COVID-19 management is focused on the specific organisational skills needed to control the outbreak. However, little is being said about generic organisational skills as the basis of specific skills and how they should be realised. Hence, the focus of this article is on crisis management through the lens of five critical generic organisational skills that give healthcare systems an opportunity to grow and thrive amid the COVID-19 pandemic.

\section{Developing Antifragility Through Generic Skills}

VUCA is a military term used to describe situations characterised by volatility, uncertainty, complexity and ambiguity. ${ }^{5}$ Disasters and crises are deemed to have a VUCA nature. In the disruptive crisis environment of VUCA, some systems become fragile and breakdown under pressure, while others remain robust and, relatively, indifferent to change. Yet, there are systems that take the opportunity of the disaster to grow and develop their antifragility amid the crisis. Many systems choose to adapt and survive, but antifragile ones evolve, grow and thrive, becoming more resilient in the future. ${ }^{6}$ The following discussion briefly presents an example of five generic organisational skills that give healthcare systems a chance to boost their antifragility in a VUCA environment like the current COVID-19 crisis. These skills include leadership, teamwork, communication, system design and learning and feedback.

\section{Leadership}

Leadership is a cornerstone for any system or organisation. The whole activity of a system revolves around the leadership style manifested in that system. Leadership is not an individual task but rather a mindset that diffuses throughout the organisation. Almost all other organisational skills, whether specific or generic, are dependent in their execution on organisational leadership, and crisis management relies heavily on leadership. ${ }^{7}$
Leadership is essential to build capacity and mitigate risks during pandemics. ${ }^{2}$ The COVID-19 outbreak has shown that leaders need to act now. ${ }^{8}$ In particular, VUCA environments, like the COVID-19 pandemic, require unique leadership characteristics. Lawrence suggests a set of leadership characteristics known as VUCA Prime to be used in VUCA environments; ${ }^{5}$ these characteristics include vision, understanding, clarity and agility. To be properly prepared, leaders need to have developed these traits well ahead of a VUCA situation. This is achieved through practice and applying the correct type of leadership styles to daily/routine situations prior to a crisis.

\section{Teamwork}

How systems and organisations perform is defined by the integrative activities of their actors. Teams are the backbone of healthcare systems and teamwork is a rate-limiting factor for organisational outcomes. The execution of healthcare activities cannot be achieved without coordinated teamwork. However, a crisis is a hard test for teams as it stretches the systems' needs beyond the normal capacity of the system. Teams in healthcare systems are the source of power in the system. In fact, there is sufficient evidence that teamwork is more important than technical prowess for preventing and mitigating a major crisis or disaster in high-risk fields such as healthcare. ${ }^{9}$

Unfortunately, it is not uncommon during a crisis for some managers to strip teams of their professional autonomy and ability to cooperate with others through the piling up of structures such as procedures and formal rules. ${ }^{10}$ As systems are stretched beyond their means during a crisis, more gaps appear at critical times, and the organisational capacity for patching and stitching that prevails during routine work is no longer sufficient. Undoubtedly, teamwork has been proved essential to address concerns related to the COVID-19 pandemic, ${ }^{11}$ and systems cannot succeed in managing the crisis without good teamwork. A crisis is a time for teamwork; leaders must equip their organisations with this invaluable skill.

\section{Communication}

A single, reliable tool during a crisis has always been communication. Over several decades, communication has been recognised as a key element for successful crisis management. ${ }^{12}$ Moreover, poor communication has been repeatedly associated with crisis management failure. ${ }^{13}$ The Joint Commission in the United States asserts that "the success or failure of an EOP [Emergency 
Operations Plan] is often determined by timely access to communication that ensures the flow of critical information". ${ }^{14}$

The COVID-19 crisis has shown how effective communication is serving as a key factor in managing the pandemic through proper flow of information, handling uncertainty and fear and promoting behavioural changes in the community. ${ }^{15}$ However, leaders must realise that this critical, key element cannot be developed overnight, especially during a crisis. Instead, communication requires time and proactive efforts well in advance of the crisis in order to establish itself in the system. A crisis provides a live test for the quality of established communication strategies within an organisation. Nevertheless, it is also a lesson for future planning.

\section{System Design}

It is a fundamental concept in biology and physiology that the structure of a system determines its functions. ${ }^{16}$ Using a medical analogy, one might think of the system's structure as the anatomy and the system's processes and functions as the physiology. For any system to serve specific outcomes, it is not enough to adjust the physiological functions through enforcing new or modified processes, policies, protocols and standards of the system unless the anatomical structure of the system is fit for the intended purpose. As has been shown during the COVID-19 crisis, many healthcare systems failed as their anatomy was not made to meet the physiological adaptations needed during a crisis.

A system that is not structurally designed to meet the challenges of crisis management will not be able to cope with the stressors resulting from required functional changes during a disaster. Although it might be late to review and modify a system's design during an emergency status, how the system responds to the challenges offers invaluable insight into how the anatomical structure of the system might present a constraint to its processes and functionality. This insight should inform a better, future system designone that takes into account the needs that may arise during crisis management, and beyond.

\section{Learning and Feedback}

Social systems, such as healthcare, are informationbonded. They change their behaviour in response to information flow within the system. ${ }^{17}$ Hence, understanding the centrality of learning and feedback in such systems is massive as it provides the means for the system to adapt to a changing external, as well as internal, environment. The quality of embedded learning and feedback functionality within a system determines, to a large extent, the quality of change in response to a dynamic environment.

The VUCA nature of reality during any crisis precludes the ability to forecast much of the changes and prepare for them in advance. In such a dynamic and constantly unfolding environment, Meadows once said: "We can't control systems or figure them out. But we can dance with them!". ${ }^{18}$ Responding within such a dynamic environment requires consciously planned and continuously monitored learning and feedback loops that allow the system to self-organise its behaviour as it emerges within the crisis.

\section{The Way Forward}

Disasters and crises disrupt the 'normal' activities of systems and organisations. However, amid this disruption, crises also offer organisations the potential to grow and thrive. The current COVID-19 pandemic is a good example of such a potential. As much as healthcare systems focus on their specific organisational skills to mitigate the effects of the crisis, one must also pay attention to the generic organisational skills through which they can develop and improve. By focusing on both specific and generic organisational skills, systems will not only survive through the crisis but will also grow and thrive towards antifragility. Antifragility and thriving are, therefore, a reflection of how much we have invested in our generic organisational skills through leadership, teamwork, communication, system's design, and learning and feedback. Systems will, almost always, output during a crisis whatever inputted in them at routine times.

\section{References}

1. Taleb NN. Antifragile: How to live in a world we don't understand. London, UK: Allen Lane, 2012.

2. Global Preparedness Monitoring Board. A world at risk. From: https://apps.who.int/gpmb/assets/annual_report/GPMB_ annualreport_2019.pdf Accessed: May 2020.

3. Global Health Security Index. Global Health Security Index October 2019. From: www.ghsindex.org/wp-content/ uploads/2020/04/2019-Global-Health-Security-Index.pdf Accessed: May 2020.

4. Friedman ML. Everyday crisis management. 1st ed. Naperville, Illinois, USA: First Decision Press, 2002.

5. Lawrence, K. Developingleaders in a VUCA environment. From: www.emergingrnleader.com/wp-content/uploads/2013/02/ developing-leaders-in-a-vuca-environment.pdf Accessed: May 2020.

6. Alhir, SS. The Anti-Fragility Edge: Antifragility in Practice. London, UK: LID Publishing, 2017.

7. Hawley A, Matheson J. Making Sense of Disaster Medicine: A Hands-on Guide for Medics. 1st ed. Boca Raton, Florida, USA: CRC Press, 2010. 
8. Fraher, AL. Thinking through crisis: Improving teamwork and leadership in high-risk fields. 1st ed. Cambridge, UK: Cambridge University Press, 2011.

9. Gates B. Responding to Covid-19 - A once-in-a-century pandemic? N Engl J Med 2020; 382:1677-9. https://doi.org/10.1056/NEJM p2003762.

10. Morieux Y, Tollman P. Six simple rules: How to manage complexity without getting complicated. Boston, Massachusetts, USA: Harvard Business Review Press, 2014.

11. Ling L, Joynt GM, Lipman J, Constantin JM, Joannes-Boyau O. COVID-19: A critical care perspective informed by lessons learnt from other viral epidemics. Anaesth Crit Care Pain Med 2020; 39:163-6. https://doi.org/10.1016/j.accpm.2020.02.002.

12. Garnett JL, Kouzmin A. Communicating throughout Katrina: Competing and complementary conceptual lenses on crisis communication. Public Adm Rev 2007; 67: 171-88. https://doi. org/10.1111/j.1540-6210.2007.00826.x.
13. Cheng SS. Crisis communication failure: A case study of typhoon Morakot. Asian Soc Sci 2013; 9:18-32. https://doi.org/10.5539/ ass.v9n3p18.

14. The Joint Commission, Joint Commission Resources. Emergency management in health care: An all-hazards approach. 2nd ed. Illinois, USA: Joint Commission Resources, 2012.

15. Finset A, Bosworth H, Butow P, Gulbrandsen P, Hulsman RL, Pieterse $\mathrm{AH}$, et al. Effective health communication - A key factor in fighting the COVID-19 pandemic. Patient Educ Couns 2020; 103:873-6. https://doi.org/10.1016/j.pec.2020.03.027.

16. Heulitt MJ. Structure determines function. J Pediatr Intensive Care 2013; 2:1-3. https://doi.org/10.3233/PIC-13041.

17. Gharajedaghi J. Systems thinking: Managing chaos and complexity: A platform for designing business architecture. 3rd ed. Burlington, Massachusetts, USA: Morgan Kaufmann, 2011.

18. Meadows DH. Thinking in systems: A primer. Hartford, Vermont, USA: Chelsea Green Publishing, 2008. 